\title{
QED effects induced harmonics generation in extreme intense laser foil interaction
}

\author{
J Y Yu ${ }^{1,4}$, T Yuan ${ }^{1,4}$, W Y Liu', M Chen ${ }^{1,4, *}$, W Luo ${ }^{2,3, *}$, \\ S M Weng ${ }^{1,4}$ and $\mathrm{Z}$ M Sheng ${ }^{1,3,4}$
}

'Key Laboratory for Laser Plasmas (MOE), School of Physics and Astronomy, Shanghai Jiao Tong University, Shanghai, 200240, China

${ }^{2}$ School of Nuclear Science and Technology, University of South China, Hengyang 421001, China

${ }^{3}$ SUPA, Department of Physics, University of Strathclyde, Glasgow G4 ONG, UK ${ }^{4}$ Collaborative Innovation Center of IFSA (CICIFSA), Shanghai Jiao Tong University, Shanghai 200240, China

*Email:minchen@sjtu.edu.cn; ${ }^{\dagger}$ Email:wenluo-ok@163.com

\begin{abstract}
A new mechanism of harmonics generation (HG) induced by quantum electrodynamics (QED) effects in extreme intense laser foil interaction is found and investigated by Particle-in-Cell (PIC) simulations. When two laser pulses with identical intensities of $1.6 \times 10^{24} \mathrm{~W} / \mathrm{cm}^{2}$ are counter-incident on a thin foil target, harmonics emission is observed in their reflected electromagnetic waves. Such harmonics radiation is excited due to transversely oscillating electric currents coming from the vibration of QED effect generated $e^{-} e^{+}$pairs. The effects of laser intensity and polarization were studied. By distinguishing the cascade depth of generated photons and pairs, the influence of QED cascades on harmonics generation was analyzed. Although the current $\mathrm{HG}$ is not an efficient way for radiation source applications, it may provide a unique way to detect the QED processes in the near future ultra-relativistic laser solid interactions.
\end{abstract}

Keywords: PIC simulation, laser plasma interaction, QED plasma

\section{Introduction}

Quantum electrodynamics (QED) has been known as a fundamental theory since the early $20^{\text {th }}$ century. The electron-positron pair $\left(e^{-} e^{+}\right.$pair) creation via photon decay was one of the most relevant topics in QED [1-3]. The development of ongoing strong laser facilities, such as ELI and Vulcan, with potential capability of delivering laser pulses in intensity of $\sim 10^{23} \mathrm{~W} / \mathrm{cm}^{2}$, makes it possible to demonstrate pair creation via laser-plasma interaction $[4,5]$. At such intensities, two important QED processes are mostly concerned. One is nonlinear Compton scattering, which describes that electrons can emit $\gamma$-ray photons when interacting with intense laser pulses [6]. Another is the well-known nonlinear Breit-Wheeler (BW) process, which describes the $e^{-} e^{+}$pair production during the interaction of high-energy $\gamma$ photons with laser photons [7]. The combination of these two processes can easily lead to a rapid growth of newly produced hard photons and $e^{-} e^{+}$pairs. This is 
known as QED cascade, or avalanche [1-3, 8, 9]. QED cascade is determined by a Lorentz invariant parameter $\chi_{i}=\left(e \hbar / m_{e}^{3} c^{4}\right) \sqrt{-\left(F_{\mu \nu} p_{i}^{v}\right)^{2}}$, where $i$ is the particle index; $\hbar$ is the reduced Plank constant; $e$ and $m_{e}$ are the elementary charge and electron mass; $F_{\mu \nu}$ and $p_{i}^{v}$ are the field tensor and particle's four-momentum. In some cases, $\chi_{i}$ can be interpreted as $\left(\varepsilon_{i} / m_{e} c^{2}\right)\left(F_{\perp} / e E_{S}\right)$, where $\varepsilon_{i}$ is the particle energy and $F_{\perp}$ is the force perpendicular to the particle's moving direction [10]. Here $E_{S}$ represents Schwinger field amplitude $E_{S}=m_{e}^{2} c^{3} / e \hbar=1.32 \times 10^{16} \mathrm{~V} / \mathrm{cm} \mathrm{[11]}$. The quantum effects come into sight when $\chi_{i}$ reaches 0.1 or even higher.

Recent researches on QED cascade effects in laser-produced-plasmas mainly concern about the pair production rate [12-14] and the energy spectra of newly generated photons and pairs [15-17]. Vranic et al. [18] and Ridgers et al. [19] have done some deep analysis on the effects of stochastic nature in the energy spectra. Different laser configurations and target shapes have been considered to lower the intensity threshold of QED cascade $[2,20]$, or to get prolific pair production [1, 3, 21-24]. Previous studies have shown that two counter-propagating lasers interacting with a foil target can form a standing wave and charged particles are hence trapped in the nodes or antinodes $[25,26]$ of the standing wave depending on the laser intensity. The electron dynamics of radiative trapping is conducted in a recent work by Kirk et al. [27]. Experimental evidences for measuring strong QED effects, especially the radiation reaction force in all-optical experiments are carried out by Poder et al. [28] and Cole et al. [29]. However, the QED effects on the reflected laser fields during the interaction, such as harmonics generation, have not been reported yet. For lower laser intensity, laser solid target interaction induced high-order harmonics generations (HHG) have been widely studied in the last decades due to their potential applications in ultraviolet- or X-ray source generation, in attosecond dynamics detection and even in plasma surface detection [30-32]. Generally, such HHGs are resulted from the electrons' classical response to the driver laser pulse, and are explained mainly by three kinds of mechanisms, i.e. relativistic oscillating mirror, coherent wake emission and coherent synchrotron emission [33-36].

In this paper, we present a new kind of harmonics generation in a regime dominated by strong QED effects, where harmonics with odd orders are observed. The mechanism of the harmonics emission is investigated by a fully relativistic Particle-in-Cell (PIC) code-EPOCH [37, 38], where two QED modules describing nonlinear Compton scattering and multi-photon BW processes are self-consistently included. Other QED processes such as BH and trident are not considered due to their relatively low cross section within current laser intensity range [39-41]. The laser polarization and intensity effects on the HGs are also studied. This mechanism may be used as a useful approach to detect and explore the QED processes in the near future ultra-relativistic laser solid interactions.

\section{Simulation setup}

In our studies, a hydrogen-carbon mixed target was used, with each atomic density of $40 n_{C}$, and the total electron density is $280 n_{C}$, where $n_{C}=\left(m_{e} \omega_{0}^{2}\right) /$ 
$\left(4 \pi e^{2}\right)$ is the critical plasma density, and $\omega_{0}$ is the laser angular frequency. Two-dimensional PIC simulations were performed. The simulation box locates from $x=0 \mu \mathrm{m}$ to $x=9 \mu \mathrm{m}$ in the longitudinal direction and from $y=-4 \mu \mathrm{m}$ to $y=4 \mu \mathrm{m}$ in the transverse direction. The target is $1 \mu \mathrm{m}$ thick and locates in the middle of the simulation box as shown in Fig. 1(a). Although the thickness we used here is not related to the relativistic skin depth, the QED process here requires that the target should not be too thick. Two counter-propagating $p$-polarized laser beams are incident simultaneously on the target. Each of them has a wavelength of $\lambda_{0}=1 \mu \mathrm{m}$ and intensity of $1.6 \times 10^{24} \mathrm{~W} / \mathrm{cm}^{2}$, which is a typical threshold intensity for QED cascade saturation $[42,43]$. Both laser pulses have a longitudinally square-pulse shape and their duration is $30 \mathrm{fs}\left(\sim 9 \mathrm{~T}_{0}\right)$. A super-Gaussian like transverse profile in $y$ direction is used for the laser pulses.

To deeply understand the QED cascade process, we made some modifications in the EPOCH code [44]. A new parameter of macroparticles called generation order is added to the particle type to describe the cascade depth of the particles, which records how a newly born particle comes from. The generation order of initial target electrons in the plasma is $G_{e}=0$. Then the generation order of photons emitted by these electrons is $G_{p}=G_{e}+1$. Afterwards, the generation order of $e^{-} e^{+}$particles is $G_{e^{-+}}=G_{p}$, etc. This process continues until the cascade stops. This is a useful diagnosis when we need to investigate the cascade effects, such as the capability of photon production from either target electrons or $e^{-} e^{+}$pairs. In our notation, all the photons with generation number of one are emitted by target electrons, meanwhile photons with generation number higher than one are emitted by the afterwards produced $e^{-} e^{+}$pairs.

\section{Harmonics generation induced by QED effects}
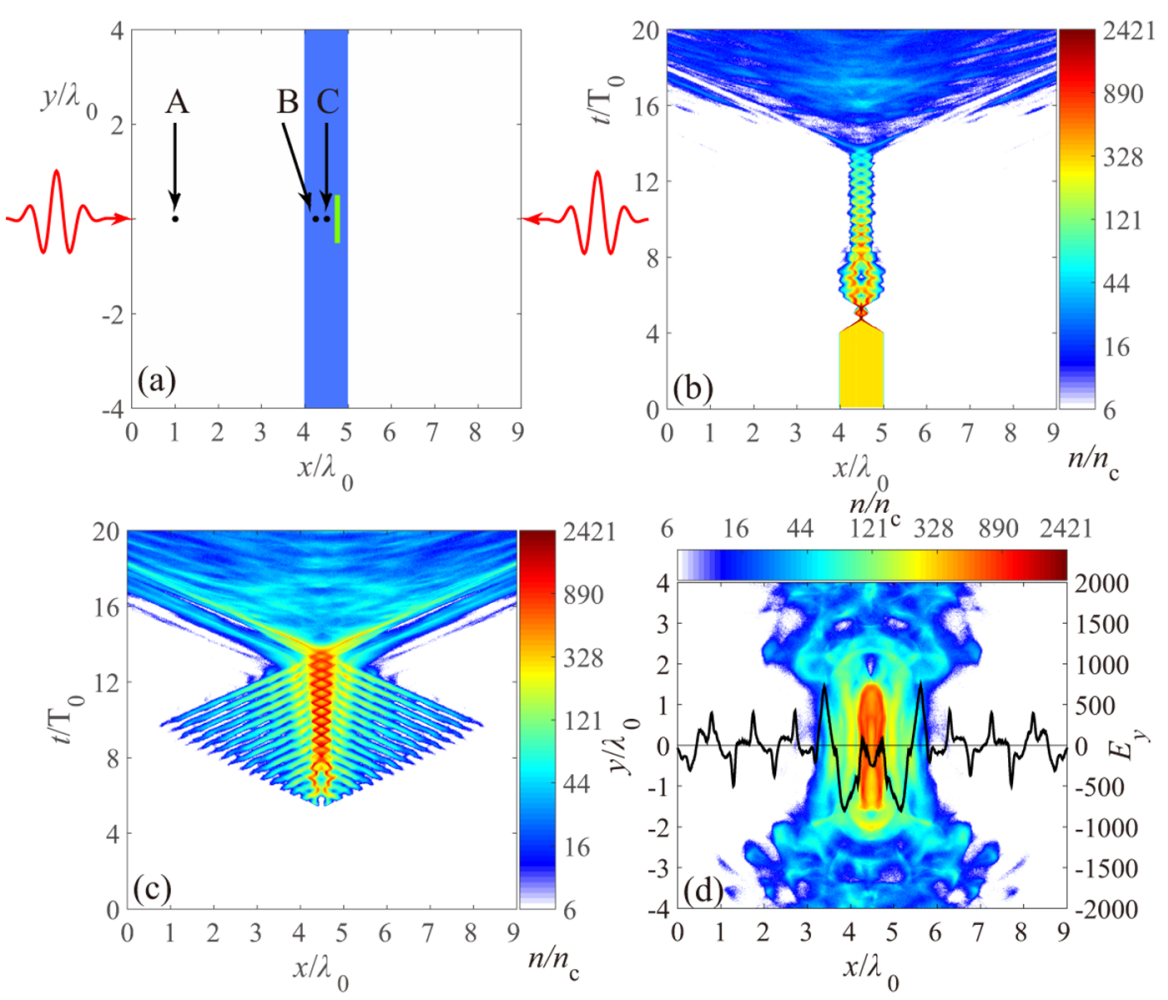
Figure 1. (a) Sketch map of the laser foil interaction and diagnosis points of the harmonics emission. The coordinates of $\mathrm{A}, \mathrm{B}$ and $\mathrm{C}$ points are $(1,0),(4.25,0)$ and $(4.5,0)$ respectively. The green region, locating from $4.7 \mu \mathrm{m}$ to $4.8 \mu \mathrm{m}$ in $x$ direction and from $-0.5 \mu \mathrm{m}$ to $0.5 \mu \mathrm{m}$ in $y$ direction, is used to diagnose the positron average density in Section 5. (b)-(c) Spatiotemporal evolutions of target electron (b) and positron (c) densities on $y=0$ axis. (d) Spatial distribution of positron density at $t=12.5 \mathrm{~T}_{0}$ and normalized electric field $E_{y}$ on $y=0$ axis.

We first study the QED process during the laser foil interaction. Fig. 1(b) and (c) show the spatiotemporal evolutions of electron and positron densities, respectively. The target electrons are firstly accelerated and compressed by lasers from both sides. Then they start thermal expansions and oscillate periodically in the laser fields, which radiate a large number of high-energy $\gamma$-photons. Due to the opposite, equal laser radiation pressures, these accelerated target electrons can be well confined to the center without any leaks, forming two high-density electron layers. The multi-photon $\mathrm{BW}$ process initiates at $t \sim 6 \mathrm{~T}_{0}$ and a large amount of $e^{-} e^{+}$pairs are therefore produced within the initial foil region. As interaction processes, $e^{-} e^{+}$pairs are produced tremendously even in the outer regions, as seen in Fig. 1(c). This is caused by the significant QED cascade effect, where the produced $\gamma$-photons propagate outwards and then collide with the incoming laser pulses, leading to continuous increase of pair plasma density. At a later time, the pair plasma becomes opaque to the incident laser pulses and the standing wave formed directly by the counter-propagating laser pulses disappears [45, 46]. Meanwhile, the electron and positron bunches in the outer regions are compressed inward by the laser ponderomotive forces which continuously increase the density of pair plasmas in the center. After $t=14 \mathrm{~T}_{0}$, the compressed pair plasma expands along both transverse and longitudinal directions due to the vanishing incident laser pulses.

The formation of the central double layer structure has also been reported by Chang et al. [47] and Baumann et al. [48], however, its effects on reflected electromagnetic (EM) fields have not been studied in detail yet. In our studies, we found that the central over dense pair plasma will introduce harmonics component in the reflected wave of incident pulses. To see this, the spatial distribution of the transverse electric field $E_{y}$ is shown in Fig. 1(d). Harmonic components can be clearly seen from the waveform. While reducing the laser intensities to $I=4 \times$ $10^{23} \mathrm{~W} / \mathrm{cm}^{2}$, the simulation shows that such kind of harmonic components disappear, which means that the current HG is quite different from what one usually observes in normal relativistic laser plasma interactions. It should be related to the QED effects. 

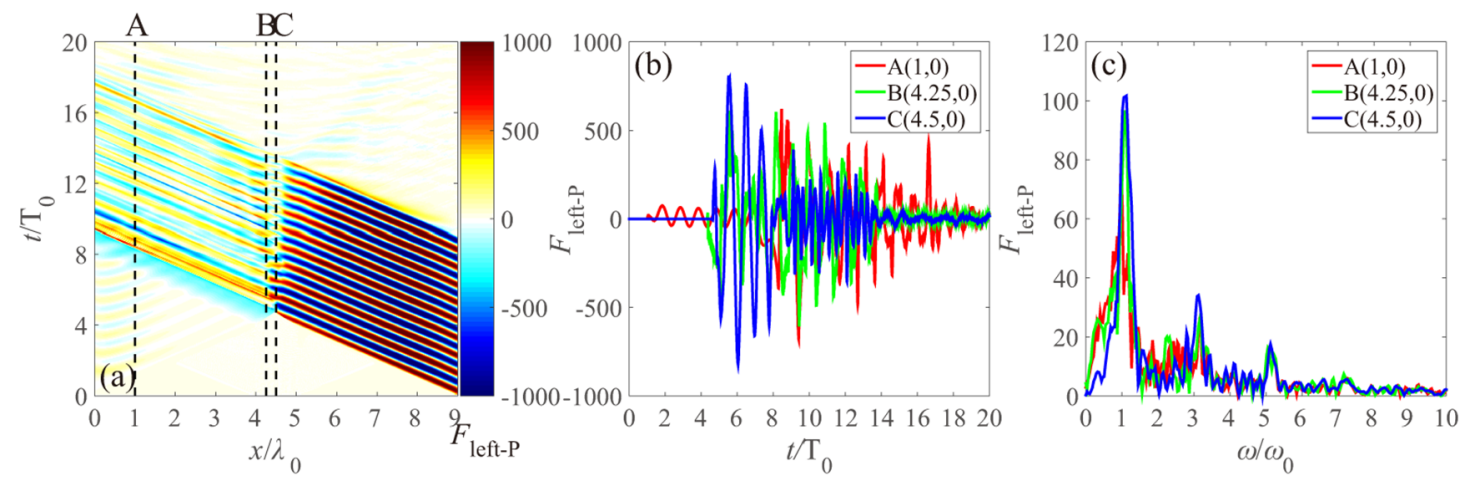

Figure 2. (a) Spatiotemporal evolution of left propagating EM field $\left(\left(E_{y}-B_{z}\right) / 2\right)$ on $y=0$ axis. Dashed lines denote the three detection points in Fig. 1. (b) Temporal evolutions of left propagating fields at detection points A, B and C. (c) Spectra of left propagating fields detected at those points.

To see the HG process, in Fig. 2(a) we show the spatiotemporal evolution of the left propagating field, which is defined as $F_{\text {left }-P}=\left(E_{y}-B_{z}\right) / 2$, where $E_{y}$ and $B_{z}$ represent the transverse electric and magnetic fields. It can be seen that most of the laser pulse cannot transmit through the high-density plasma layers formed in the central region. In specific, temporal evolutions of left propagating fields and their Fast Fourier Transformation (FFT) at the three detection points are shown in Fig. 2(b) and (c), respectively. One can see the $3^{\text {rd }}$ and $5^{\text {th }}$ harmonic components at all these detection points, which is consistent with the spatial distribution of the fields shown in Fig. 1 (d). From Fig. 2(b) one can see that the initial reflected or transmitted waves only contain fundamental frequency components and the high order component appears at a later interaction stage, which implies that the HG happens as the QED process is fully excited. In the following we demonstrate the effect of QED cascades on the harmonics generation by changing laser foil interaction parameters, such as laser polarization and intensity.

\section{Laser polarization and intensity effects on harmonics generation}

We first study the laser polarization effect on harmonics generation. While keeping $p$-polarization of the left incident beam, the polarization of the right incident beam is changed to $s$-polarization (We call this P-S driven interaction case). By this way one can distinguish whether the harmonics come from the transmission or the reflection component of each laser pulse. 

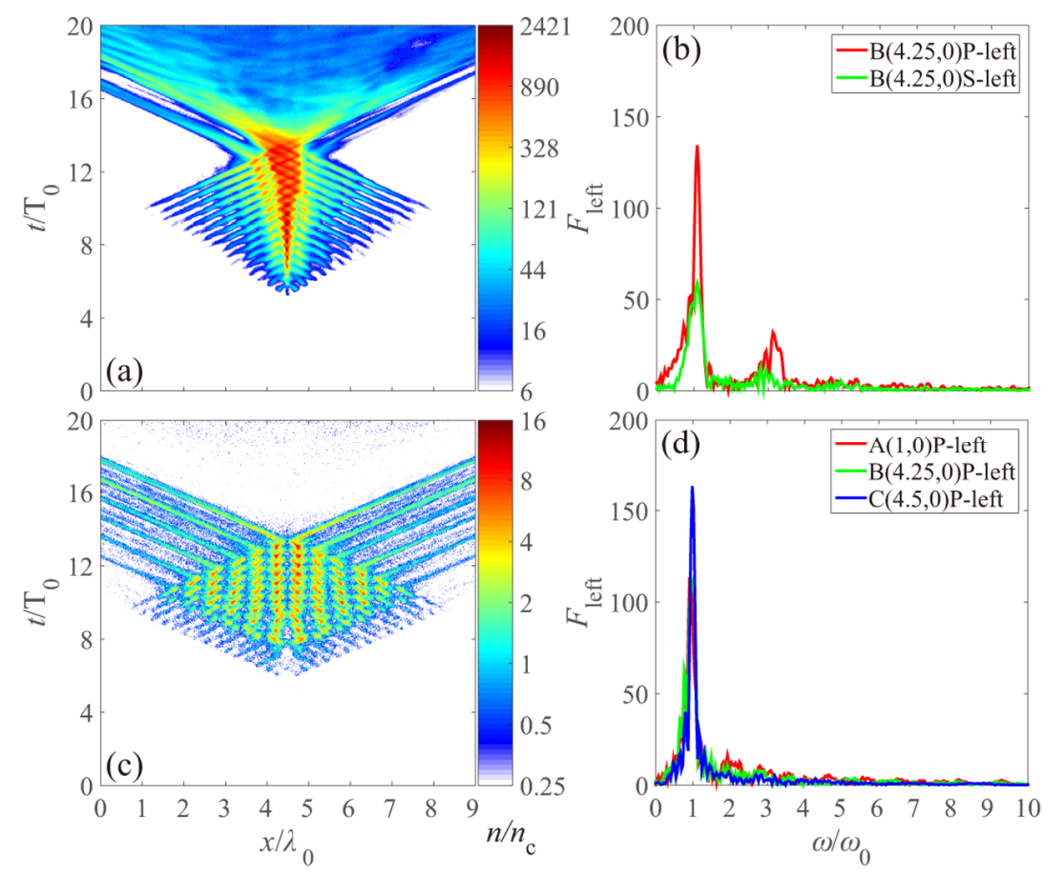

Figure 3. (a, c) Spatiotemporal evolution of positron density on $y=0$ axis. (b, d) Spectra of different filed components at three detection points. Upper and lower pads correspond to the P-S driver interactions at laser intensity of $1.6 \times 10^{24} \mathrm{~W} / \mathrm{cm}^{2}$ and $\mathrm{P}-\mathrm{P}$ driver interactions at laser intensity of $4 \times 10^{23} \mathrm{~W} / \mathrm{cm}^{2}$, respectively.

We present in Fig. 3(a), the spatiotemporal evolution of positron density of the $\mathrm{P}-\mathrm{S}$ driver interaction case. It is seen that the left propagating $s$-polarized laser seems to have a stronger force on the produced pair plasma leading to a tilted distribution of positron density. This is due to a quasi-1D effect of the $s$-polarization in a 2D-slab simulation structure. The particles cannot move along the $z$ direction such that they accumulate around the interaction region, which enhances the production of $e^{-} e^{+}$ pairs. The FFT spectra of left propagating $p / s$-polarized field components at point B are shown in Fig. 3(b). Here, the left propagating $s$ component field is defined as $F_{\text {left-s }}=\left(E_{z}+B_{y}\right) / 2$. Both left propagating $p$-polarized component and $s$-polarized component have a peak in the $3^{\text {rd }}$ harmonic. The $p$ component is larger than the $s$ component, which means the harmonics mainly come from the reflected EM waves.

The effect of laser intensity on harmonics generation is further studied. We reduced the laser intensity to $4 \times 10^{23} \mathrm{~W} / \mathrm{cm}^{2}$. The spatiotemporal evolution of positron density and the FFT spectra of the propagating EM fields are shown in Fig. 3(c) and (d), respectively. The positron density decreases with the laser intensity. The central high-density plasma layer disappears and the sanding wave stays during the whole interaction process. The FFT spectra show that the $3^{\text {rd }}$ and $5^{\text {th }}$ harmonics components no longer exist at all of the three detection points, and the amplitude of the fundamental frequency wave becomes higher relative to the case of $I=1.6 \times$ $10^{24} \mathrm{~W} / \mathrm{cm}^{2}$. This is mainly due to the weaker laser absorption at the lower laser intensity.

\section{QED cascade effects on harmonics generation and source of electric currents}




\section{inducing the harmonics emission}
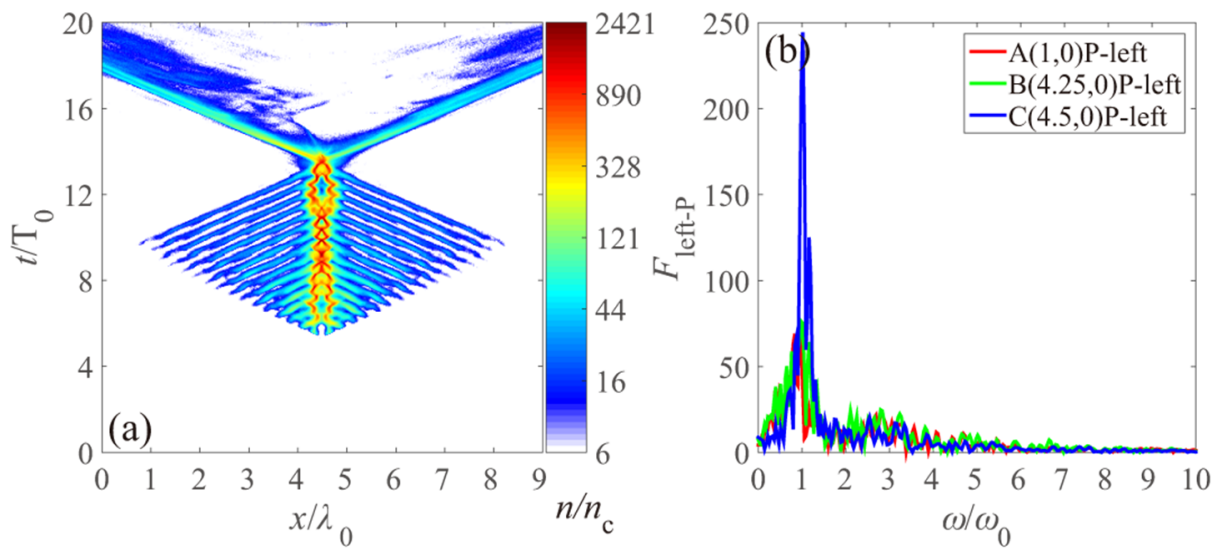

Figure 4. (a) Spatiotemporal evolution of positron density on $y=0$ axis for the case without QED cascade process. (b) FFT spectra of left propagating fields at points A, B and $\mathrm{C}$.

In the high-density regime, QED cascades play an important role in the production of dense pair plasmas. To further check the effect of the QED cascade process on the harmonics generation, in a simulation we turned off the QED cascade channel. We only allow the production of the first generation of $\gamma$ photons. The generation of $e^{-} e^{+}$pairs from the laser target electrons interaction reserves, however, the succeeding cascade process is switched off. So technically the photons with $G_{p} \geq 2$ do not generate $e^{-} e^{+}$pairs (see also in Fig. 5(e)). In this case, the pair number as well as its spatial density has a sharp decrease as shown in Fig. 4(a). Accordingly, the density oscillation of the two QED plasma layers becomes much weaker, which further reduces the laser absorption and reflection. Figure 4(b) shows the FFT spectra of left propagating fields at the three detection points. It is seen that all the harmonics components are vanished and only some high frequency noise remains in the spectra. The peak value of the fundamental frequency is much higher than that obtained in the cascade-turn-on case, which means that most of the laser energy do not transfer to the target plasmas and the $e^{-} e^{+}$pairs. Beside the lower density of the oscillating electrons, by comparing Fig. 4(a) and Fig. 1(c), one can also see that the oscillation pattern itself is not as stable as the one in the QED cascade on case. These two factors make the conventional HHG mechanisms (such as relativistic oscillation mirror mechanism) be almost absent in current laser plasma interaction region. It proves that the harmonics generation here is mainly governed by the QED cascades and only happens when the laser intensity becomes strong enough, which may provide a useful way to detect the QED processes. 

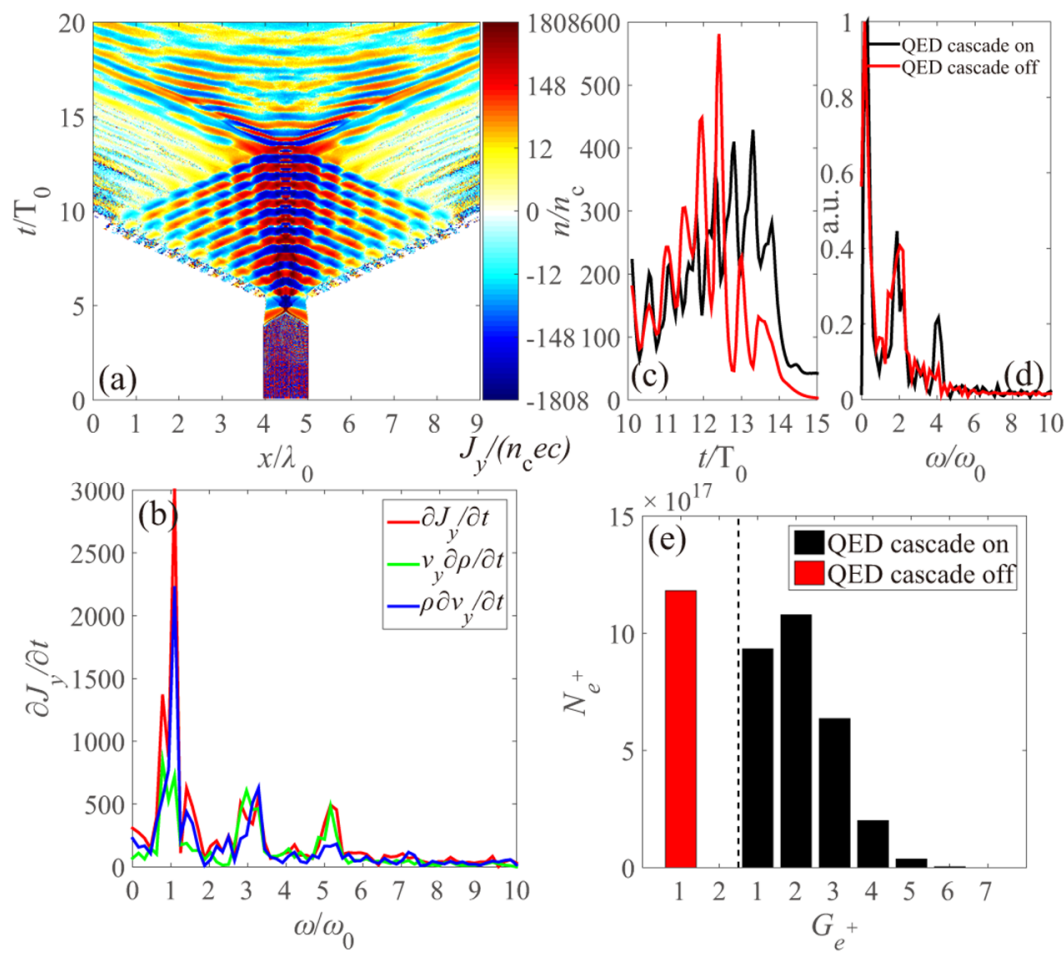

Figure 5. (a) Spatiotemporal evolution of transverse electric current $J_{y}$ on $y=0$ axis. (b) FFT spectra of $\partial J_{y} / \partial t$ and its two contribution terms for the harmonics generation. The contribution terms are averaged within the green square region labelled in Fig. 1(a). (c) Temporal evolution of average positron density within the green square region labelled in Fig. 1(a) for the cases with QED cascade on (black line) and off (red line). (d) FFT of the corresponding lines in (c). (e) Generation order spectra of positrons for the cases with the QED cascade on (black squares) and off (red squares) at $t=12.5 \mathrm{~T}_{0}$. The QED cascade is turned on for the figures (a) and (b).

To investigate the mechanism of the harmonics generation in the current study, we checked the transverse electric current evolution in the PIC simulations. Figure 5 (a) shows the spatiotemporal evolution of $J_{y}$ on $y=0$ axis. It is found that from about $t=8 \mathrm{~T}_{0}$ harmonics components begin to appear in the central area. The FFT spectra of the current derivative $\partial J_{y} / \partial t$ and its two contribution terms, $v_{y} \partial \rho / \partial t$ and $\rho \partial v_{y} / \partial t$ are shown in Fig. 5(b). One can see that in this QED cascade on case, the $v_{y} \partial \rho / \partial t$ term has a dominated contribution to the $5^{\text {th }}$ harmonic component.

Since the frequency component of $v_{y}$ is mainly one $\omega$, it means the density derivative term should include $4 \omega$ component. This is confirmed in Fig. 5(c), where evolutions of the average positron density in the green square region labelled in Fig. 1(a) for the QED cascade on and off cases are shown. Before $t=10 \mathrm{~T}_{0}$ the evolutions for the two cases show similar behavior. A double-frequency $(2 \omega)$ component of $\rho$ is seen in both cases as shown in Fig. 5(d), which is mainly due to the $2 \omega$ component of the longitudinal ponderomotive force of the driver lasers. Combined with the one $\omega$ component evolution of $v_{y}$, both of the two terms 
( $v_{y} \partial \rho / \partial t$ and $\rho \partial v_{y} / \partial t$ ) contribute to the $3^{\text {rd }}$ component peak in Fig. 5(b).

From the black lines in Fig. 5(c) and (d) one can also see that besides the $2 \omega$ component, in the QED cascade on case, a $4 \omega$ component of $\rho$ also exists.

Combined with the one $\omega$ component evolution of $\partial v_{y} / \partial t, \rho \partial v_{y} / \partial t$ term gives the $5^{\text {th }}$ component peak in Fig. 5(b). And this electric current component finally contributes to the $5^{\text {th }}$ harmonic in the electromagnetic spectrum.

To find the reason of the $4 \omega$ component of $\rho$, we used our newly developed function of cascade order analysis. Figure 5 (e) shows the positron generation orders in the QED cascade on and cascade off cases. When QED cascade is artificially turned off, positrons with $G_{e^{+}} \geq 2$ no longer exist and as a result, the total positron number in this case is much lower than that in the QED cascade on case. This also explains why the average positron number in the QED cascade off case decreases much quickly than the QED cascade on case after $\mathrm{t}=12.5 \mathrm{~T}_{0}$. The simulation results show that the $4 \omega$ component of pair density oscillation only appears when the cascade process is turned on. It might because that the pairs with higher generation number $\left(G_{e^{ \pm}} \geq 2\right)$ depend on the first generation of pairs $\left(G_{e^{ \pm}}=1\right)$ whose density modulation have $2 \omega$ component. Combining with the $2 \omega$ component contribution from the interacting lasers, the final pair density modulations show $4 \omega$ component, which then leads to the generation of the $5^{\text {th }}$ order current and harmonic as we mentioned before.

\section{Summary and discussion}

In summary, the dynamics of two ultra-relativistic intense counter-propagating lasers interacting with a thin foil target is studied by using QED module included PIC simulations and harmonics up to $5^{\text {th }}$ order have been observed. It shows that such harmonics are generated due to QED effects, especially the cascade process. Both the transmission and reflection fields carry harmonics components, while the reflection carries majority. Once QED cascade effect is turned off artificially or by reducing the driver laser intensity, $e^{-} e^{+}$pairs production will be suppressed and harmonics component disappears. These studies provide a new way to detect the QED effects in the coming extreme intense laser target interaction where the QED processes may dominate the interaction.

\section{Acknowledgements}

This work was supported by the National Basic Research Program of China (Grant No. 2013CBA01504) and the National Science Foundation of China (Grant Nos. 11421064, 11374209, and 11374210). Simulations were performed on the $\Pi$ Supercomputer at Shanghai Jiao Tong University and Tianhe II supercomputer at Guangzhou.

\section{References}

[1] Bell A R and Kirk J G 2008 Phys. Rev. Lett. 101200403

[2] Fedotov A M, Narozhny N B, Mourou G and Korn G 2010 Phys. Rev. Lett. 105 
080402

[3] Nerush E N, Kostyukov I Y, Fedotov A M, Narozhny N B, Elkina N V and Ruhl H 2011 Phys. Rev. Lett. 106035001

[4] Extreme light infrastructure, http://www.eli-laser.eu/

[5] The vulcan 10 petawatt project, https://www.clf.stfc.ac.uk/Pages/

Vulcan.aspx

[6] Di Piazza A, Hatsagortsyan K Z and Keitel C H 2010 Phys. Rev. Lett. 105220403

[7] Breit G and Wheeler J A 1934 Phys. Rev. 461087

[8] Landau L and Rumer G 1938 Proc. R. Soc. London A 166925

[9] Bulanov S, Narozhny N, Mur V and Popov V 2006 J. Exp. Theor. Phys. 1029

[10] Di Piazza A, Muller C, Hatsagortsyan K Z and Keitel C H 2012 Rev. Mod. Phys. 841177

[11] Schwinger J 1951 Phys. Rev. 82664

[12] Elkina N V, Fedotov A M, Kostyukov I Y, Legkov M V, Narozhny N B, Nerush E N and RuhlH 2011 Phys. Rev. Spec. Top. Accel. Beams 14054401

[13] Tang S, Bake M A, Wang H Y and Xie B S 2014 Phys. Rev. A 89022105

[14] Kostyukov I Y and Nerush E N 2016 Phys. Plasmas 23093119

[15] Ji L L, Pukhov A, Nerush E N, Kostyukov I Y, Shen B F and Akli K U 2014 Phys. Plasmas 21023109

[16] Nerush E, Kostyukov I, Ji L and Pukhov A 2014 Phys. Plasmas 21013109

[17] Nerush E N and Kostyukov I Y 2015 Plasma Phys. Control. Fusion 57035007

[18] Vranic M, Grismayer T, Fonseca R A and Silva L O 2016 New J. Phys. 18 073035

[19] Ridgers C P et al 2017 J. Plasma Phys. 83715830502

[20] Gelfer E G, Mironov A A, Fedotov A M, Bashmakov V F, Nerush E N, Kostyukov I Y and Narozhny N B 2015 Phys. Rev. A 92022113

[21] Bashmakov V F, Nerush E N, Kostyukov I Y, Fedotov A M and Narozhny N B 2014 Phys. Plasmas 21013105

[22] Zhu X L, Yin Y, Yu T P, Shao F Q, Ge Z Y, Wang W Q and Liu J J 2015 New J. Phys. 17053039

[23] Zhu X L, Yu T P, Sheng Z M, Yin Y, Turcu I C E and Pukhov A 2016 Nat. Commun. 713686

[24] Liu J J, Yu T P, Yin Y, Zhu X L and Shao F Q 2016 Opt. Express 24 15978-15986

[25] Gonoskov A, Bashinov A, Gonoskov I, Harvey C, Ilderton A, Kim A, Marklund M, Mourou G and Sergeev A 2014 Phys. Rev. Lett. 113014801

[26] Jirka M, Klimo O, Bulanov S V, Esirkepov T Z, Gelfer E, Bulanov S S, Weber S and Korn G 2016 Phys. Rev. E 93023207

[27] Kirk J G 2016 Plasma Phys. Control. Fusion 58085005

[28] Poder K et al 2018 arXiv:1709.01861v3 [physics.plasm-ph]

[29] Cole J M et al 2018 arXiv:1707.06821v2 [physics.plasm-ph]

[30] Dromey B et al 2006 Nat. Phys. 2 456-459

[31] Wheeler J A, Borot A, Monchoce S, Vincenti H, Ricci A, Malvache A, Lopez-Martens R and Quere F 2012 Nat. Photon. 6 829-833

[32] Leblanc A, Monchoce S, Bourassin-Bouchet C, Kahaly S and Quere F 2016 Nat. 
Phys. 12 301-305

[33] Baeva T, Gordienko S and Pukhov A 2006 Phys. Rev. E 74046404

[34] Quere F, Thaury C, Monot P, Dobosz S, Martin P, Geindre J P and Audebert P 2006 Phys. Rev. Lett. 96125004

[35] Dromey B et al 2012 Nat. Phys. 8 804-808

[36] Zhang G B, Chen M, Liu F, Yuan X H, Weng S M, Zheng J, Ma Y Y, Shao F Q, Sheng Z M and Zhang J 2017 Opt. Express 25 23567-23578

[37] Arber T D et al 2015 Plasma Phys. Control. Fusion 57113001

[38] Ridgers C P, Kirk J G, Duclous R, Blackburn T G, Brady C S, Bennett K, Arber

T D and Bell A R 2014 J. Comput. Phys. 260 273-285

[39] Liang E P, Wilks S C and Tabak M 1998 Phys. Rev. Lett. 814887

[40] Liu J X et al 2015 Phys. Plasmas 22103102

[41] Burke D L et al 1997 Phys. Rev. Lett. 791626

[42] Fedotov A, Narozhny N and Mironov A 2016 J. Phys.: Conf. Ser. 691012023

[43] Luo W, Liu W Y, Yuan T, Chen M, Yu J Y, Li F Y, Del Sorbo D, Ridgers C P and Sheng Z M 2017 (submitted)

[44] Yuan T, Yu J Y, Liu W Y, Weng S M, Yuan X H, Luo W, Chen M, Sheng Z M and Zhang J 2017 (submitted)

[45] Liu W Y, Luo W, Yuan T, Yu J Y, Chen M and Sheng Z M 2017 Phys. Plasmas 24 103130

[46] Luo W, Zhu Y B, Zhuo H B, Ma Y Y, Song Y M, Zhu Z C, Wang X D, Li X H, Turcu I C E and Chen M 2015 Phys. Plasmas 22063112

[47] Chang H X, Qiao B, Xu Z, Xu X R, Zhou C T, Yan X Q, Wu S Z, Borghesi M, Zepf M and He X T 2015 Phys. Rev. E 92053107

[48] Baumann C and Pukhov A 2016 Phys. Rev. E 94063204 